\title{
FINITELY PRESENTED GROUPS OF FIBONACCI TYPE. PART II
}

\author{
COLIN M. CAMPBELL and EDMUND F. ROBERTSON
}

(Received 8 March; revised 15 August 1978)

Communicated by $\mathbf{H}$. Lausch

\begin{abstract}
A class of cyclically presented groups with $n$ generators and $n$ Fibonacci type relations is discussed. Conditions are given for these groups to be finite and metacyclic. With these conditions the presentations are reduced to the standard form for metacyclic groups with trivial Schur multiplicator. This enables certain isomorphisms between the groups to be found.
\end{abstract}

Subject classification (Amer. Math. Soc. (MOS) 1970): 20 F 05.

\section{Introduction}

Groups with symmetrical presentations, called cyclically presented groups, have been the subject of investigation in recent years. In this paper we continue work on cyclically presented groups which was begun in Campbell and Robertson (1975b).

A group is said to be cyclically presented if it has a presentation on $n$ generators $a_{1}, a_{2}, \ldots, a_{n}$ with $n$ relations obtained from a single word $w=w\left(a_{1}, a_{2}, \ldots, a_{n}\right)$ by permuting the subscripts modulo $n$ according to the powers of the permutation $(12 \ldots n)$. The resulting group is denoted by $G_{n}(w)$. The interest in cyclically presented groups most relevant to this paper arises from the study of Fibonacci groups, these being the groups $\mathrm{F}(r, n)=G_{n}(w)$ where $w=a_{1} a_{2} \ldots a_{r} a_{r+1}^{-1}$. A survey of results on Fibonacci groups and other cyclically presented groups is contained in Johnson, Wamsley and Wright (1974) and Chalk and Johnson (1977), see also Johnson (1976), Chapter 16 and Lyndon and Schupp (1977), Chapter 2. In Johnson, Wamsley and Wright (1974) the groups $\mathbf{F}(r, n)$, with $r \equiv 1 \bmod n$, are shown to be finite metacyclic groups and one of the problems posed in that article is to find standard two generator presentations for these metacyclic groups. This problem 
is solved in Campbell and Robertson (1975a). For integers $r, n, k$ and $s$ with $r>s \geqslant 1 ; n \geqslant 1, k \geqslant 0$ define the class of groups $\mathbf{F}(r, n, k, s)$ by $\mathbf{F}(r, n, k, s)=G_{n}(w)$ where $w=a_{1} a_{2} \ldots a_{r}\left(a_{r+k} a_{r+k+1} \ldots a_{r+k+s-1}\right)^{-1}$.

In this paper we examine metacyclic groups in the class $\mathbf{F}(r, n, k, s)$ giving presentations in standard two generator form. As a corollary the presentation problem is solved for the class $\mathbf{F}(r, n, 1, s)$ studied in Campbell and Robertson (1975b). We shall, where possible, use the notation and results of Campbell and Robertson (1975b). The authors would like to thank the referee for encouraging them to produce a more readable paper.

\section{A two generator two relation presentation}

All finite metacyclic groups may be presented in the form

$$
\left\langle a, b \mid a^{M}=1, b^{-1} a b=a^{R}, b^{N}=a^{T}\right\rangle
$$

where $R^{N} \equiv 1 \bmod M$ and $T=L M /(M, R-1)$ for some non-zero integer $L$, see Zassenhaus (1958), p. 129. Beyl (1973) shows that the finite metacyclic groups with trivial Schur multiplicator are precisely those which may be given by the presentation (2.1) with $L=1$. Now $\mathrm{F}(r, n, k, s)$, when finite, has deficiency zero and so has trivial Schur multiplicator. Hence, in order to apply Beyl's result, we aim to reduce the presentation for $\mathrm{F}(r, n, k, s)$ to the form (2.1) when $r, n, k$ and $s$ satisfy certain conditions.

We begin by obtaining two generator two relation presentations for the groups $\mathbf{F}(r, n, k, s)$ under slightly more general conditions than we shall need for our main result. These more general conditions are $r \equiv s \bmod n,(r, n)=1,(r+k-1, n)=1$ and we shall assume that these hold for the rest of this section. Our first step towards producing a two generator presentation of $\mathbf{F}(r, n, k, s)$ is to obtain another $n$-generator presentation and to do this we require the following notation.

Let $[i]$ denote the integer $i$ reduced modulo $n$ so that $0 \leqslant[i]<n$ and define $t$ and $u$ by $t=(r-[r]) / n, u=(s-[s]) / n$. For any integer $i$ not congruent to 1 modulo $n$ let $b_{i}=a_{1} a_{2} \ldots a_{[i-1]}$; also define $b_{1}=1$ and $x=a_{1} a_{2} \ldots a_{n}$. Further define $\varepsilon_{i}$, for any integer $i$, by

$$
\varepsilon_{i}= \begin{cases}1 & \text { if }[i-1]+[r] \geqslant n, \\ 0 & \text { otherwise }\end{cases}
$$

Now the $j$ th relation

becomes

$$
a_{j} a_{j+1} \ldots a_{j+r-1}=a_{j+r+k-1} a_{j+r+k} \ldots a_{j+r+k+s-2}
$$

$$
b_{j}^{-1} x^{l+\varepsilon_{j}} b_{j+r}=b_{j+r+k-1}^{-1} x^{u+\varepsilon_{j+r+k-1}} b_{j+2 r+k-1}
$$


We now show that $x$ and $y=b_{r+k}$ generate $\mathbf{F}(r, n, k, s)$ and then find a presentation using these generators. Given any $j$ there is an $i$ with $j \equiv i r+1 \bmod n$ so we may define $c_{j}$ by

$$
c_{i r+1}=b_{(i+1) r+k} b_{i r+1}^{-1} .
$$

Now (2.2) with $j=r+1$ gives

$$
c_{i r+1}=x^{u+\varepsilon_{(i+1) r+k}} c_{(i+1) r+1} x^{-\left(l+\varepsilon_{i r+1}\right)} .
$$

Since $(r, n)=1$ and $c_{1}=b_{r+k} b_{1}^{-1}=y$ we see, from (2.4), that $c_{j} \in\langle x, y\rangle$ for all $j$. Now, since $(r+k-1, n)=1$, (2.3) shows that $b_{j} \in\langle x, y\rangle$ for all $j$ and hence $\langle x, y\rangle=\mathrm{F}(r, n, k, s)$. Again using $c_{1}=y$ together with repeated use of (2.4) we obtain the relation

$$
y=x^{-p} y x^{q}
$$

where $p=\sum_{i=1}^{n}\left(t+\varepsilon_{i r+1}\right)$ and $q=\sum_{i=1}^{n}\left(u+\varepsilon_{(i+1) r+k}\right)$. Since $(r, n)=1$ this gives

$$
y^{-1} x^{s} y=x^{r} \text {. }
$$

Only one further non-trivial relation in $x$ and $y$ is obtained for $F(r, n, k, s)$. Substituting for the $c$ 's in $\prod_{i=1}^{n} c_{i(1-r-k)+1}$ their expressions given by $(2,3)$ shows that

$$
\prod_{i=1}^{n} c_{i(1-r-k)+1}=1 \text {. }
$$

We introduce further notation to enable (2.6) to be written as a relation in $x$ and $y$. Define $\lambda$ to be $\left[r^{-1}(r+k-1)\right]$. Let $p_{1}=\varepsilon_{1}+\varepsilon_{1+r^{-1}}+\varepsilon_{1+2 r^{-1}}+\ldots+\varepsilon_{k}$ and, for $1<i \leqslant n$, define

$$
p_{i}= \begin{cases}p_{1}-[r] & \text { if there exists an integer } j \text { with }(i-1) \lambda<j n \leqslant i \lambda, \\ p_{1} & \text { otherwise. }\end{cases}
$$

Also let $\beta(i)=[(n-i+1) \lambda] t-[(n-i) \lambda] u+p_{n-i+1}$. Now $c_{i(r+k-1)+1}=c_{i \lambda r+1}$. Therefore (2.4) together with $c_{1}=y$ gives

$$
c_{i(r+k-1)+1}=x^{-a(i)} y x^{b(i)}
$$

where

$$
a(i)=[i \lambda] u+\sum_{j=1}^{[i \lambda]} \varepsilon_{j r+k} \quad \text { and } \quad b(i)=[i \lambda] t+\sum_{j=1}^{[i \lambda]} \varepsilon_{1+(j-1) r}
$$

Substituting (2.7) into (2.6) gives, after some elementary calculation,

$$
\prod_{i=1}^{n}\left(x^{\beta(i)} y\right)=1 .
$$

We have proved the following theorem. 
THEOREM 1. Suppose that
(i) $r \equiv s \bmod n$,
(ii) $(r, n)=1$,
(iii) $(r+k-1, n)=1$.

Then $\mathbf{F}(r, n, k, s)$ has the two generator two relation presentation

$$
\left\langle x, y \mid y^{-1} x^{s} y=x^{r}, \prod_{i=1}^{n}\left(x^{\beta(i)} y\right)=1\right\rangle .
$$

Corollary.

$$
\mathbf{F}(r, n, 1, s)=\left\langle x, y \mid y^{-1} x^{s} y=x^{r}, x^{r}=\prod_{i=1}^{n}\left(x^{(n-i+1) t-(n-i) u} y\right)\right\rangle
$$

where $r \equiv s \bmod n$ and $(r, n)=1$.

Notice that with $a=x, b=y x^{b}$ we obtain from this corollary the presentation for $\mathbf{F}(r, n, 1, s)$ given in Theorem 2 of Campbell and Robertson (1975b).

\section{A standard metacyclic Schur group presentation}

In this section we shall assume that the conditions $r \equiv s \bmod n,(r, s)=1$ and $(r+k-1, n)=1$ hold. Note that these conditions imply $(r, n)=1$ and so the results of Section 2 are valid. We shall now show how, under these conditions, the group $\mathbf{F}(r, n, k, s)$ may be given by the presentation (2.1) and so, by the comments at the beginning of Section 2, as a standard presentation for a metacyclic Schur group. (A Schur group is a group with trivial Schur multiplicator.) Put $\sigma=r^{n}-s^{n}$. Since $r$ and $s$ are coprime $(\sigma, s)=1$ and we may define $\alpha$ to be the inverse of $s$ modulo $\sigma$. Although the next two lemmas are not stated explicitly in Campbell and Robertson (1975b) they can readily be deduced from the proof of Theorem 3 of that paper.

LEMMA 1. The relations

$$
y^{-1} x^{s} y=x^{r} \quad \text { and } \quad x^{r}=\prod_{i=1}^{n}\left(x^{(n-i+1) t-(n-i) u} y\right)
$$

together imply

$$
y^{-1} x y=x^{\alpha r} \text { and } y^{n}=x^{\eta}
$$

where $\eta$ is such that

$$
(1-\alpha r) \eta \equiv\left(\frac{s-r}{n}\right)\left(\frac{1-(\alpha r)^{n}}{1-\alpha r}\right) \bmod \sigma .
$$


LEMMA 2.

$$
\left(\sigma,\left(\frac{r-s}{n}\right)\left(\frac{1-(\alpha r)^{n}}{1-\alpha r}\right)\right)=\frac{\sigma}{n}
$$

It turns out that (2.8) can be expressed in a more suitable form. Indeed the next three results are concerned with doing just this.

LEMMA 3. The relation $\prod_{i=1}^{n}\left(x^{\beta(i)} y\right)=1$ may be written in the form

$$
x^{\lambda r}=\left(\prod_{i=1}^{n}\left(x^{(n-i+1) \lambda t-(n-i) \lambda u} y\right)\right) x^{\mu},
$$

where $\mu \equiv\left(p_{1}\left(1-(\alpha r)^{n}\right) /(1-\alpha r)\right) \bmod \sigma$.

Proof. Let $\gamma_{i}=(i \lambda-[i \lambda]) / n$. Then

$$
\begin{aligned}
\beta(n-i+1) & =i \lambda t-\gamma_{i} n t-(i-1) \lambda u+\gamma_{i-1} n u+p_{i} \\
& =(i \lambda t-(i-1) \lambda u)-\gamma_{i} r+\gamma_{i-1} s+p_{1}
\end{aligned}
$$

Now $\gamma_{1}=\gamma_{0}=0$ and $\gamma_{n-1}=\lambda-1$ and so (2.8) becomes

$$
\left(x^{\lambda u-n+p_{1}-[r]} y\right)\left(\prod_{i=2}^{n}\left(x^{(n-i+1) \lambda t-(n-i) \lambda u} x^{d(i)} y\right)\right)=1
$$

where $d(i)=-\gamma_{n-i+1} r+\gamma_{n-i} s+p_{1}$. If we take each of the expressions $x^{d(i)}$ to the right hand side of (3.1) using the relation $y^{-1} x y=x^{\alpha r}$ we obtain

$$
x^{\lambda r}=\left(\prod_{i=1}^{n}\left(x^{(n-i+1) \lambda(-(n-i) \lambda u} y\right)\right) x^{\mu},
$$

where $\mu=\left(\sum_{i=1}^{n-1}\left(-\gamma_{i} r+\gamma_{i-1} s+p_{1}\right)(\alpha r)^{i}\right)+(\lambda-1) s+p_{1}$. To complete the proof we simplify $\mu$ working modulo $\sigma$. Since $\alpha s \equiv 1$ we have $\gamma_{i-1} \alpha s r \equiv \gamma_{t-1} r$ and so

$$
\mu \equiv-\gamma_{n-1} r(\alpha r)^{n-1}+p_{1} \sum_{i=0}^{n-1}(\alpha r)^{i}+(\lambda-1) s .
$$

But $(\lambda-1) s \equiv(\lambda-1) r(\alpha r)^{n-1}$ since both $(\alpha r)^{n} \equiv 1$ and $\alpha s \equiv 1$. Therefore

$$
\mu \equiv p_{1} \sum_{i=0}^{n-1}(\alpha r)^{i}
$$

as required.

Using the same method as in Lemma 1 we can reduce (2.8) to $y^{n}=x^{v}$ for some $\nu$. Therefore $y^{n}$ is central and, since $y^{-n} x^{8^{n}} y^{n}=x^{r^{n}}, x^{\sigma}=1$.

LEMMA 4. $\nu \equiv(\lambda \eta-\mu) \bmod \sigma$. 
Proof. By Lemma 1

$$
x^{\lambda r}=\prod_{i=1}^{n}\left(x^{(n-i+1) \lambda \ell-(n-i) \lambda u} y\right)
$$

may be written as

$$
y^{n}=x^{\lambda \eta}
$$

Substituting into (3.2) gives $y^{n}=x^{\lambda \eta-\mu}$ as required.

LEMMA 5. $(1-\alpha r) \mu \equiv 0 \bmod \sigma$.

Proof. This follows immediately using the expression for $\mu$ given in Lemma 3 since $1-(\alpha r)^{n} \equiv 0 \bmod \sigma$.

We are now in a position to find the standard form presentations.

THEOREM 2. Suppose that
(i) $r \equiv s \bmod n$,
(ii) $(r, s)=1$,
(iii) $(r+k-1, n)=1$.

Then $\mathbf{F}(r, n, k, s)$ is a finite metacyclic Schur group with presentation

$$
\left\langle a, b \mid a^{\sigma / n}=1, b^{-1} a b=a^{\alpha r}, b^{n}=a^{\sigma / n(r-s)}\right\rangle .
$$

Proof. The order of the generator $x$ is $((1-\alpha r) \nu, \sigma)$. But

$$
\begin{aligned}
((1-\alpha r) \nu, \sigma) & =((1-\alpha r) \lambda \eta-(1-\alpha r) \mu, \sigma) & & \text { by Lemma } 4, \\
& =((1-\alpha r) \lambda \eta, \sigma) & & \text { by Lemma } 5, \\
& =\left(\left(\frac{r-s}{n}\right)\left(\frac{1-(\alpha r)^{n}}{1-\alpha r}\right), \sigma\right) & & \text { by Lemma } 1, \\
& =\sigma / n & & \text { by Lemma } 2 .
\end{aligned}
$$

Also $\nu$ is divisible by $\sigma / n(s-r)$. For

$$
\begin{aligned}
\nu & \equiv(\lambda \eta-\mu) \bmod \sigma / n(s-r) & & \text { by Lemma } 4, \\
& \equiv \lambda \eta \bmod \sigma / n(s-r) & & \text { by Lemma } 5, \\
& \equiv 0 \bmod \sigma / n(s-r) & & \text { by Lemma } 1 .
\end{aligned}
$$

Hence relation (2.8) becomes

for some integer $\xi$.

$$
y^{n}=x^{\xi \sigma / n(8-r)}
$$


Therefore, when conditions (i), (ii) and (iii) are satisfied, $\mathbf{F}(r, n, k, s)$ has the presentation

$$
\left\langle x, y \mid x^{\sigma / n}=1, y^{-1} x y=x^{\alpha r}, y^{n}=x^{\xi \sigma / n(8-r)}\right\rangle .
$$

The fact that $(\alpha r)^{n} \equiv 1 \bmod \sigma / n$ now shows that the results of Beyl (1973) are applicable to $\mathbf{F}(r, n, k, s)$ when $r, n, k$ and $s$ satisfy conditions (i), (ii) and (iii) and so it may be presented in the form

$$
\left\langle a, b \mid a^{\sigma / n}=1, b^{-1} a b=a^{\alpha r}, b^{n}=a^{\sigma / n(r-8)}\right\rangle .
$$

Finally, as a consequence of the $k$ independence of the presentation given in Theorem 2, we have the following corollary.

Corollary. Suppose that

(i) $r \equiv s \bmod n$, (ii) $(r, s)=1$, (iii) $(r+k-1, n)=1$, (iv) $\left(r+k^{\prime}-1, n\right)=1$. Then $\mathrm{F}(r, n, k, s)$ is isomorphic to $\mathbf{F}\left(r, n, k^{\prime}, s\right)$.

\section{References}

F. R. Beyl (1973), 'The Schur multiplicator of metacyclic groups', Proc. Amer. Math. Soc. 40, 413-418.

C. M. Campbell and E. F. Robertson (1975a), 'On metacyclic Fibonacci groups', Proc. Edinburgh Math. Soc. 19, 253-256.

C. M. Campbell and E. F. Robertson (1975b), 'On a class of finitely presented groups of Fibonacci type', J. London Math. Soc. 11, 249-255.

C. P. Chalk and D. L. Johnson (1977), 'The Fibonacci groups II', Proc. Roy. Soc. Edinburgh 77A, 79-86.

D. L. Johnson (1976), Presentations of groups (Cambridge University Press, Cambridge).

D. L. Johnson, J. W. Wamsley and D. Wright (1974), 'The Fibonacci groups', Proc. London Math. Soc. 29, 577-592.

R. C. Lyndon and P. E. Schupp (1977), Combinatorial group theory (Springer, Berlin).

H. Zassenhaus (1958), The theory of groups (Chelsea, New York, 2nd ed.).

The Mathematical Institute

University of St. Andrews

St. Andrews KY16 9SS

Scotland 